\title{
Horizontal Vertical Diagonal Gabor Binary Pattern Descriptor with PLDA for Pose-Invariant Face Recognition
}

\author{
Kumud Arora \\ Department of Computer Science and Engineering, Inderprastha Engineering College, Ghaziabad, INDIA \\ Email:kumud.arora76@gmail.com \\ Poonam Garg \\ Department of Information Technology, Institute of Management \& Technology, Ghaziabad, INDIA \\ Email:pgarg@imt.edu
}

Received: 19 July 2018; Accepted: 17 September 2018; Published: 08 December 2018

\begin{abstract}
Face recognition is one of the conventional problems in computer vision. Its recognition rate falls steeply when the images are captured in an unconstrained environment. One of the fundamental issues that creep into unconstrained environment capturing is that of the face pose variation. Due to face pose variation, occlusion of crucial features takes place. Occlusion may lead to information loss in the face descriptor which describes the face appearance. In this paper, we propose learningbased descriptor that combines horizontal, vertical and diagonal pattern of blocks generated from the convolution of face image with Gabor filter bank. To use only discriminative features, Probabilistic Linear Discriminant Analysis (PLDA) is used. The fusion of non-uniform texture based descriptor along with the PLDA approach aids in retaining enough of discriminative information to overcome the information loss occurring during feature occlusion. Since HVDGBP face descriptor utilizes the fundamental concept of Linear Binary Pattern (LBP) henceforth it helped in meeting low processing demands and ease of computing characteristic required for good face descriptors. Comprehensive comparative performance analysis of the robustness for the proposed face descriptor to withstand pose variations is presented. UMIST and AT\&T Database is used for experimental analysis.
\end{abstract}

Index Terms-Face Pose Recognition, Probabilistic Linear Discriminant Analysis (PLDA), Gabor Linear Binary Pattern, Pose Recognition.

\section{INTRODUCTION}

Reliable face recognition is motivated not only by the essential challenges this classical recognition-problem poses but also by the issues that creep in during its various practical applications where unconstrained face identification is needed. Face recognition is among the core biometric technologies whose applications are growing leaps and bounds due to their significant role in security, surveillance and forensics. Among the conventional steps (face pre-processing, feature extraction, feature matching and feature classification) followed for face recognition, feature extraction is one of the crucial steps. Feature extraction provides information desired for distinguishing between faces of different persons. If the reliable feature set is extracted, then there is increase in the probability of obtaining all relevant information from the input data and henceforth improvement in the recognition rate. However, out of the plethora of face descriptors defined in the literature, only a few can resist the changes in face pose. The primary issue with feature descriptors for pose images is that they not only encode desired subject information but they also encode unwanted pose related information. Whereas, a robust face feature descriptor is expected to retain the subject details intact even though the face has undergone pose transformation like translation, rotation or scaling.

Face descriptors may be categorized based on their application. Descriptors may describe the face appearance holistically or patch-wise, incorporating features of the whole face or a set of features of facial components and thereby comprising dense or sparse descriptors. Descriptors may be data dependent or data independent. Based on learning, learning descriptors may be categorized into shallow learning descriptors or deep learning descriptors. Learning descriptors evolve their characteristics by learning from the training data. Deep learning descriptors often involve a massive volume of the dataset from where the descriptor is to be determined, and deep convolutional networks (CNN's) are used for the learning process. Though, deep learning descriptors delivers impressive performance but still their processing demands are very high. On the other side, easy to compute face descriptor-intensity based descriptor fails completely when the pose variations are present. 

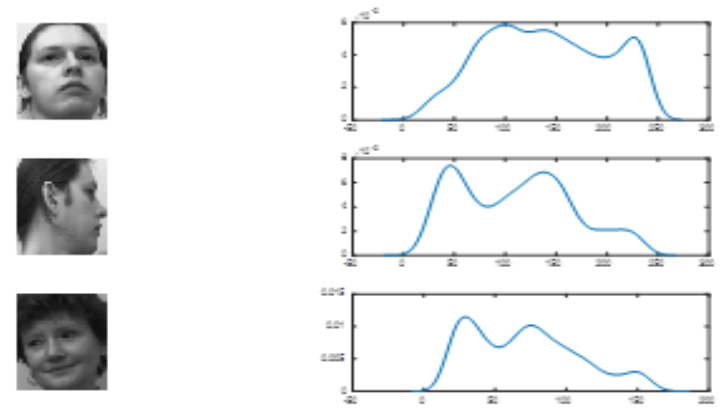

Fig.1. Intensity Descriptor of Same and Different Subject

Their failure may be accounted for considering only appearance information rather than semantic information. During pose change face appearance changes. Fig.1 clearly shows the effect on intensity distribution due to pose change. It can be deduced that intensity distribution undergoes change to such an extent that it may resemble more with the intensity distribution of a different subject rather than its own subject.

Recently, texture based local descriptors have gained attention due to its low computational demands. However, the presence of pose variation restricts one to contemplate a large local neighborhood. In this paper, for the pose invariant feature extraction, non-uniform texture based descriptor: Horizontal Vertical Diagonal Gabor Pattern (HVDGBP) descriptor is proposed by utilizing linear binary pattern and Gabor filters. To reduce the dimensionality of HVDGBP descriptor, Local Linear Embedding (LLE) is utilized. Probabilistic Linear Discriminant Analysis (PLDA) is utilized to boost the discriminative power of dimensionality reduced descriptor. In section two, the related work to LBP based descriptor, Gabor filter based descriptor and PLDA is presented. Section three presents the proposed approach while section four presents the experimental results and analysis of the proposed descriptor. Conclusion and future works are discussed in the Conclusion section.

\section{RELATED WORKS}

This section delves into the face descriptors based on Linear Binary Pattern and Gabor filters. A brief review of PLDA is also presented, as it was used to select discriminatory feature set based on the probabilistic framework.

\section{A. LBP-Based Face Descriptors}

One of the most extensively used local descriptors is the linear binary pattern descriptor proposed by Ojala, Pietikäinen and Harwood [1]. The idea of using LBP is driven by the fact that faces can be seen as a composition of micro-patterns. LBP descriptor describes the local facial texture units by calculating the intensity differences and then encoding the differences of the $3 \times 3$ neighborhood. Of $3 \times 3$ pixel block, the center pixel of is assigned a value equal to the sum of the encoded values for the differences (eq. 1).

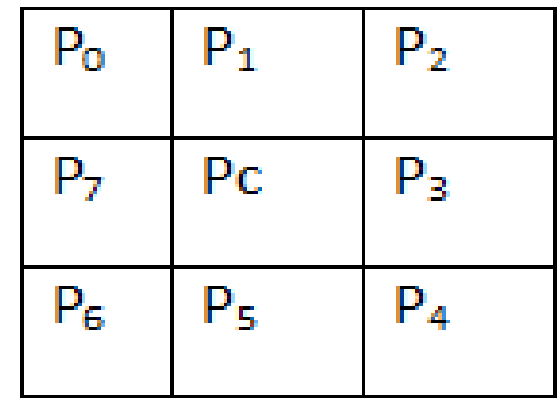

Fig.2. Sample Cell Block

$$
P_{c}=\sum_{i=0}^{7} s\left(P_{i}-P_{c}\right) \cdot 2^{i}
$$

where

$$
s(z)= \begin{cases}\mathbf{1}, \text { if } & z>=\mathbf{0} \\ \mathbf{0}, \text { if } & z<\mathbf{0}\end{cases}
$$

Centre pixel encoding of $3 \times 3$ blocks, when performed on the whole face image, tend to average over the image area. Spatially enhanced LBP calculates texture descriptors for small face region independently and then calculate the frequency occurrence pattern of encoded differences [2]. The local descriptors are concatenated to form a description of the global geometry of a face image. Spatially enhanced LBP contains information on pixel level, regional level and at complete face image itself. The size of the descriptor is $n \times m$ where $n$ is the length of the histogram and $\mathrm{m}$ is the number of regions in which face is split into.

Though LBP is easy to compute, still it is extremely prone to intensity perturbations and the pose variances therefore often lead to wrong descriptor information. Ahonen, Matas, He \& Pietikäinen [3] in their work attempted to improve the robustness of LBP by using LBP Histograms of Discrete Fourier Transforms (DFT) as face descriptor. Zhang, Gao, Zhao \& Liu [4] proposed Local Derivative Pattern (LDP) based on encoding distinctive directional pattern features. LDP denotes the higher-order derivative pattern of images with the enhanced capability to seize the more detailed discriminative information than the LBP. Trefný and Matas [5] also proposed two new encoding schemes Transition Local Binary Patterns (tLBP) and Direction coded Local Binary Pattern (dLBP), for representation of the intensity function in a local neighborhood. One of the proposed schemes dLBP possesses invariance to gray scale inversion. Huang, Shan, Ardabilian, Wang and Chen [6] surveyed the LBP based approaches for face recognition. In their survey work they highlighted the illumination variation sensitivity of LBP and stressed upon the necessity to preprocess the images before applying LBP. Recently, Dubey and Mukherjee [7] proposed a local directional order pattern (LDOP) that computes pattern for a block by finding the relationship between the center pixel and the local directional order 
indices. The histogram of LDOP is computed over the whole image to construct the descriptor. This descriptor does not depend upon the number of neighbors involved to compute the order. Rather, it depends only upon the number of directions. Hussain, Napoleon and Jurie [8] proposed a generalization of local pattern features-Linear Quantization Patterns (LQP). LQP features provided better flexibility to exploit local information thereby giving better accuracy rates. Lu, Liong, Zhou \& Zhou [9] in their work proposed a compact binary face descriptor (CBFD). With the learned feature filter, they attempted to remove the redundancy information of the original raw pixels. Tian, Fan and Ming [10] proposed iterative quantization binary codes (IQBC) - data driven binary face learning descriptor utilizing face image discriminative information. In their iterative quantization scheme, they proposed to minimize the quantization error by optimizing rotation transformation parameters that map data to the apexes of a zero-centered binary hypercube [10]. In all the mentioned works it was stressed that pre-processing of LBP is required to make it robust to illumination, noise and pose. Even after two decades, extensive research is still going on to improve the discriminative power of LBP and its robustness against local changes.

\section{B. Gabor Descriptors}

Gabor descriptors are based on Gabor filters named after Dennis Gabor. Daugman [11] showed correspondence between the 2D Gabor functions and the physiognomies of the mammalian visual system. In the spatial domain, 2D Gabor filter can be represented as Gaussian kernel function modulated by a sinusoidal plane wave, i.e.

$$
G_{\mu, v}(z)=I(z) \cdot \psi_{\mu v}(z)
$$

where $I(z)$ denotes the input image and $z$ is the pixel (x,y) which is under consideration and $\psi_{\mu, v}(\mathrm{z})$ is the Gabor kernel with orientation $\mu$, scale $v$ and is defined as

$$
\left.\psi_{\mu, v}=\frac{\left\|\mathrm{k}_{\mu, v}\right\|^{2}}{\sigma^{2}} \cdot \mathrm{e}^{\left(-\frac{\left\|\mathrm{k}_{\mu, v}\right\|^{2} \cdot\|\mathrm{z}\|^{2}}{2 \sigma^{2}}\right)} \cdot \mathrm{e}^{\mathrm{i} \mathrm{k}_{\mu v}(\mathrm{z})}-\mathrm{e}^{-\sigma^{2} / 2}\right]
$$

$\mathrm{k}_{\mu, v}$ is a wave vector and defined as $\mathrm{k}_{\mu, \mathrm{v}}=\mathrm{k}_{\mathrm{v}} \cdot \mathrm{e}^{\mathrm{i} \Phi \mathrm{u}}$ with $\mathrm{K}_{v}=\mathrm{k}_{\max } / \mathrm{f}^{\mathrm{v}} \quad\left(\mathrm{k}_{\max }\right.$ is the maximum frequency, $\mathrm{f}$ is the spacing between the kernels in the frequency domain), $\Phi \mu$ is the orientation with value $\Phi \mu=(\pi . \mu) / 8$. Gabor filter gives the peak response at the region where there is a texture change as well at the edge areas. Their response is based on spatial frequency, orientation and direction. Shen, Bai and Fairhurst [12] in their work proved that better recognition accuracy is achieved by using Gabor feature-based methods in place of raw pixels. They also proved that the discriminatory ability of Gabor features is also better as compared to other feature descriptors. Perez, Cament and Castillo [13] proposed Local Matching Gabor (LMG), based on matching the local Gabor features jets by utilizing 'Borda Count' strategy. Total computational time for LMG was very high as the total of 4172 Gabor jets were used. Gabor energy feature is utilized by joining the response of the filter to the phase pairs. This resulted in more computational complexity and higher response time. Haghighat, Zonouz and AbdelMottaleb [14] in their work proved that a set of Gabor filters with diverse frequencies and orientations is helpful in extracting useful features from an image. Combination of LBP pattern with Gabor filter resulted in extraction the non-uniform LBP Pattern from an input image [20] which can't be extracted by a uniform LBP Pattern. Recently, Jami, Chalamala, and Kakkirala [15] collectively utilized Gabor wavelet and cross local binary patterns for representing face image features along with the Probabilistic Linear Discriminant Analysis framework for tolerating pose-variations while recognition.

\section{Probabilistic Linear Discriminant Analysis (PLDA)}

LDA is equivalent to maximum likelihood classification under the assumption that each class follows Gaussian distributions. Ioffe [16] proposed "Probabilistic Linear Discriminant Analyzer" (PLDA) that reformulates problem of dimension reduction in a probabilistic context. PLDA model treats the face image as an additive sum of two parts, a deterministic part which depends on an underlying representation of identity only, and a stochastic part which explains the pose variations due to which the two face images from the same person are not identical. LDA is expressed as the maximum likelihood solution of probabilistic latent variables in PLDA, i.e., PLDA uses the latent variable model to define a generative process for each object. The latent variables characterize both the class of the object and the view of the object within the class. Considering training data to be collection of ' $\mathrm{k}$ ' images each of ' $\mathrm{N}$ ' individuals, each pose image (' $j$,th $)$ of ' $i$, th individual can be modeled as:

$$
\mathrm{x}_{\mathrm{i}, \mathrm{j}}=\mu+\text { F.h }_{\mathrm{i}}+\mathrm{G}_{\mathrm{w}} \mathrm{w}_{\mathrm{j}}+\varepsilon_{\mathrm{i}, \mathrm{j}}
$$

The signal part of model $\left(\mu+F . h_{i}\right)$ is dependent upon the subject identity (there's no dependence upon $\mathrm{j}$ ) and poses variation part $\left(\mathrm{G} . \mathrm{w}_{\mathrm{ij}}+\epsilon_{\mathrm{ij}}\right.$, ) depends upon the amount of pose variation within subject's identity. Term $\mu$ represents the mean of complete train dataset. The matrix ' $F$,' ' $G$ ' comprises the basis for a betweenindividual subspace and within individual subspace respectively. ' $\mathrm{F}$ ' matrix is approximately equivalent to the eigenvectors of the between-individual co-variance matrix and matrix ' $G$ ' is equivalent to eigenvectors of within individual covariance matrix. ' $F$ ' variable is shared by all the varied poses of the same subject. The Latent variable ' $h_{i}$ ' represents the position of the given observation within between-individual subspace (F) and ' $\mathrm{w}_{\mathrm{ij}}$ ' represent the position of the given observation within individual subspace. The term ' $\epsilon_{\mathrm{ij}}$ ' represents zero mean Gaussian-distributed noise variable with covariance $\Sigma$. Hence, the model is parameterized by $\theta=\{\mu, \mathrm{F}, \mathrm{G}, \Sigma\}$. In the training phase, set of parameters 
defined by $\theta$ are learned from training data ' $w_{\mathrm{ij}}$ '. During the recognition phase, the inference is made on the basis of these parameters. PLDA gives a principled method to combine different features in a way that more discriminative features have more impact. In standard PLDA formulation, all latent variables have a Gaussian distribution. The probability distribution of latent variables follows normal distribution i.e.

$$
\begin{gathered}
\mathrm{h}_{\mathrm{i}} \sim N(0, \mathrm{I}) \\
\mathrm{W}_{\mathrm{ij}} \sim N(0, \mathrm{I}) \\
\sim N(0, \Sigma)
\end{gathered}
$$

The distribution of observed variable can be expressed as:

$$
x_{i j} \mid h_{i,} w_{i j} \sim N\left(x_{i j} \mid \mu+F h_{i}+G w_{i j}, \Sigma\right)
$$

Sizov, Lee and Kinnunen [17] provide simple implementation of PLDA via EM algorithm. EM algorithm is utilized for estimation of model parameters. During classification, ' $\mathrm{C}$ ' models (equal to the number of classes) are built up, and the likelihood of the probe data is estimated for these models. Each model represents a relationship between latent identity variable and the latent within individual difference variable.

\section{PROPOSED APPROACH}

The proposed approach framework can be divided into four divisions:
A. Image Pre-Processing
B. Feature Extraction
C. Dimensionality Reduction
D. Probabilistic Model Building using PLDA.

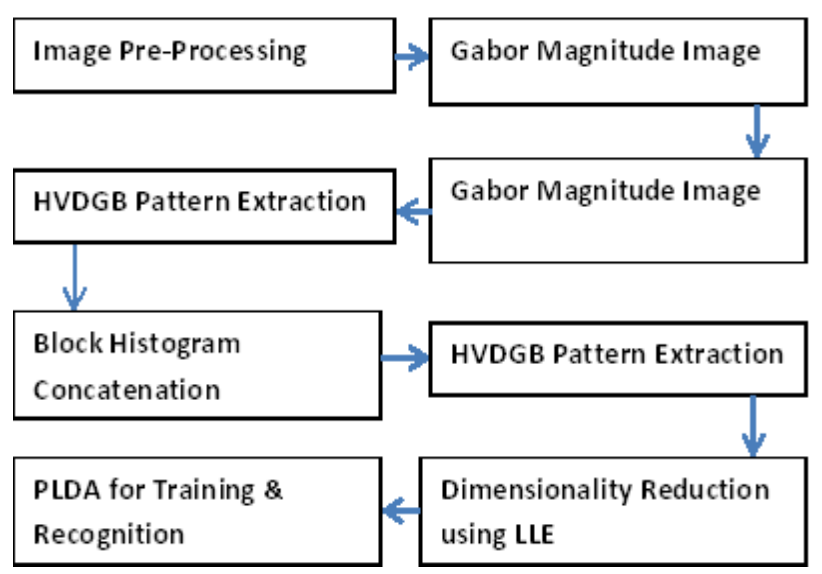

Fig.3. Framework for HVDGBP with PLDA for Face Recognition

\section{A. Image Pre-Processing}

The Image Pre-processing step consists of face detection. The cropped image undergoes image normalization so that distribution of intensity values of image follows a normal distribution. Gabor magnitude images in the frequency domain are obtained by convolving 40 Gabor filter bank of five spatial frequencies and eight orientation parameters. Magnitude values of each of the 40 images per subject are calculated, and a combined magnitude image is derived. Then a binary pattern is computed using the HVD descriptor of $5 \times 5$ non-overlapping cells.

\section{B. HVDGBP (Horizontal Vertical Diagonal Gabor Binary Pattern)}

The pattern is generated by considering immediate neighbors and extended neighbors of the cell and thresholding function's'. Gabor magnitude image is considered as input for HVDGBP. The magnitude image of the complex Gabor filter combines the sensitivity of even and odd parts of the filter into a measure of orientation's strength. Pattern is generated across $5 \times 5$ cell. For each cell ' $C$ ' represents center pixel, blue colored pixels are the pixels across vertical direction, while red colored pixels are across diagonal directions and green colored pixels are across horizontal direction of the cell. Pattern for the immediate neighboring pixels under consideration, is described by:

$$
D_{0}=\sum_{n=1}^{8} 2^{n-1} \cdot s\left(I_{n} \cdot I_{c}\right)
$$

where $I_{n}$ is the immediate neighbor block under consideration, and $I_{c}$ is the center pixel of Gabor magnitude image.

For extended neighboring pixels under consideration, the pattern is described by

$$
D_{1}=\sum_{\mathrm{n}=1}^{8} 2^{\mathrm{n}-1} \cdot \mathrm{s}\left(\mathrm{I}_{\left.8+2 . \mathrm{n}-1^{-\mathrm{I}_{\mathrm{c}}}\right)}\right.
$$

The decimal value for the two binary patterns is then averaged with round off function to convert decimal values to the integer values for assignment to the center pixel.

$$
\operatorname{HVDGP}\left(\mathrm{I}_{\mathrm{c}}\right)=\text { round }\left(\left(\mathrm{D}_{0}+\mathrm{D}_{1}\right) / 2\right)
$$

For HVDGBP generation, total sixteen logical comparisons are required for $5 \times 5$ cell, and no interpolation of values is required. Since the cell size is $5 \times 5$, for an image with dimensions that are not perfect multiples of five, two options can be taken up with one being zero padding and another being leaving the pixels of the fractional block. For this paper, the second option is considered. For an image with $92 \times 112$ size, the dimension considered for binary pattern build up is $90 \times 110$ with total $18 \times 22=396$ non-overlapping cells. For each cell, out of 25 pixels, 16 pixels are considered for the descriptor. Thus, pixel percentage under consideration 
is reduced by $36 \%$ thereby increasing the computation speed. Fig. 4 elaborates the HVDGB pattern generation.

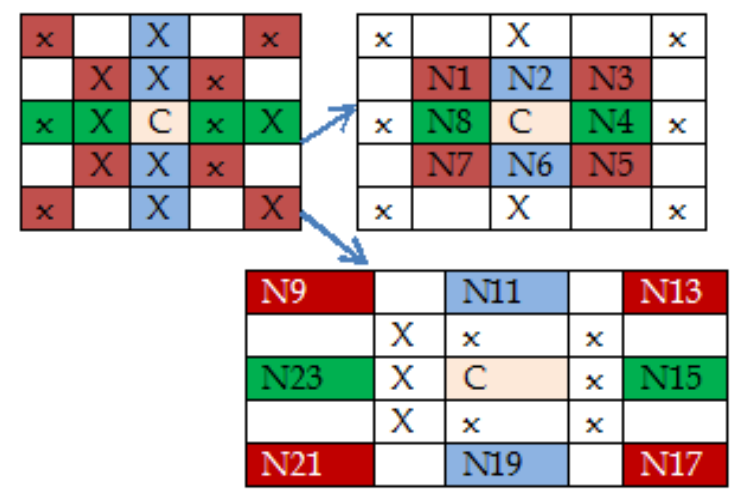

\begin{tabular}{l}
$\mathrm{D}_{0}=2^{0} \cdot s(\mathrm{~N} 1, \mathrm{C})+2^{1} \cdot s(\mathrm{~N} 2, \mathrm{C})+2^{2} \cdot s(\mathrm{~N} 3, \mathrm{C})+$ \\
$2^{3} \cdot s(\mathrm{~N} 4, \mathrm{C})+2^{4} \cdot s(\mathrm{~N} 5, \mathrm{C})+2^{5} \cdot s(\mathrm{~N} 6, \mathrm{C})+$ \\
$2^{6} \cdot s(\mathrm{~N} 7, \mathrm{C})$ \\
\hline $\mathrm{D}_{1}=2^{0} \cdot s(\mathrm{~N} 9, \mathrm{C})+2^{1} \cdot s(\mathrm{~N} 11, \mathrm{C})+2^{2} \cdot s(\mathrm{~N} 13, \mathrm{C})+$ \\
$2^{3} \cdot s(\mathrm{~N} 15, \mathrm{C})+2^{4} \cdot s(\mathrm{~N} 17, \mathrm{C})+2^{5} \cdot s(\mathrm{~N} 19, \mathrm{C})+$ \\
$2^{6} \cdot s(\mathrm{~N} 21, \mathrm{C})+2^{7} \cdot s(\mathrm{~N} 23, \mathrm{C})$ \\
\hline
\end{tabular}

$$
\mathrm{D}=\left(\mathrm{D}_{0}+\mathrm{D}_{1}\right) / 2
$$

Fig.4. HVD Pattern Extraction from the $5 \times 5$ cell

To extract the local histogram features of HVDGBP image map, concatenated sequence of histograms $\left(\mathrm{H}=\left\{\mathrm{H}_{11}, \mathrm{H}_{12}, \mathrm{H}_{13} \mathrm{H}_{14} \mathrm{H}_{15}, \mathrm{H}_{21}, \mathrm{H}_{22} \mathrm{H}_{23} \ldots \mathrm{H}_{51} \mathrm{H}_{52} \mathrm{H}_{53} \mathrm{H}_{54}\right.\right.$ $\left.\mathrm{H}_{55}\right\}$ ) is obtained from all the non-overlapping cells. ' $\mathrm{H}$ ' serve as the facial features. The local histogram extraction process is repeated for all three hundred ninety six cells. The total length of feature vector per image with eight bins per histogram is $(90 \times 110) /(5 \times 5) \times 8=3168$.

\section{Dimension Reduction and Classification}

Dimensionality reduction approaches reduces feature vector dimension to improve classification accuracy. Classification accuracy is better if the number of training images is more than the dimensionality of the feature vector. PLDA being a linear model performs best when the data obey linear assumptions. However, face-pose classification is a representative case of non-linear classification, and linear dimension reduction approaches will obviously not be able to give the desired result. LLE (Local Linear Embedding) proposed by Roweis \& Saul [18] is used for nonlinear dimension reduction. The internal structure of data is discovered by using the neighbourhood information. The input to LLE consists of feature space of length 3168 of 20 subjects with 10 train images (3168x200) and five neighbourhood points $(\mathrm{K}=5)$ per train data point are taken. Optimum dimensionality reduction value is considered after observing the impact on the equal error rate. Then the probability distribution of each class is computed independently. PLDA builds models $\theta_{\mathrm{i}=}\left\{\mu_{\mathrm{i}}, \mathrm{F}_{\mathrm{i}}, \mathrm{G}_{\mathrm{i}}, \Sigma_{\mathrm{i}}\right\}$, using latent variables that define inter-class and inter-class variation. Expectation
Maximization algorithm is used to estimate model parameters. During classification, ' $\mathrm{C}$ ' models (equal to the number of classes) are built up, and the likelihood of the probe data is estimated for these models. Each model represents a relationship between latent identity variable and the latent within individual difference variable. Figure 5 represents the constructed models as:
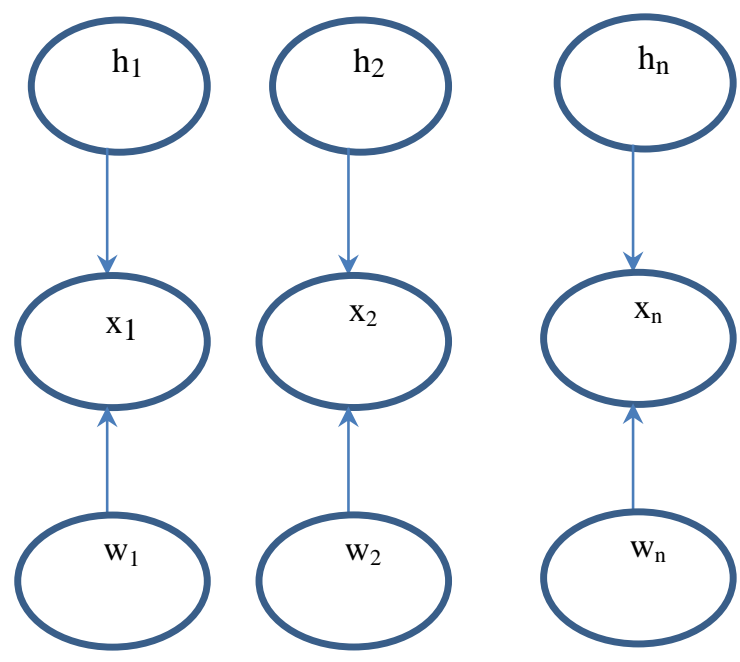

Fig.5. PLDA Model

The log-likelihood ratio is computed for $\mathrm{C}$ different

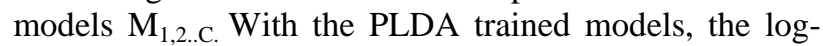
likelihood ratio is calculated. From the log-likelihood ratios, the recognition accuracy is calculated between enrolled and probe databases.

\section{Algorithm for Proposed Approach}

\section{Procedure HVDGBP_PLDA}

Inputs: Train Image Database $X \epsilon n_{1} x R^{m}$, Test Database $Y \epsilon n_{2} \times R^{n}$ partitioned intoEnrollment Database and Probe Database.

\section{Output: Array of Log-likelihood ratios}

a. Image normalization process is executed for every face image.

b. For each image in Train Database (X) \& Test Database (Y), compute face features by using HVDGBP descriptor $X^{\prime} \leftarrow \operatorname{HVDBGP}(X)$, $\mathrm{Y}^{\prime} \leftarrow \operatorname{HVDGBP}(\mathrm{Y})$

c. Dimension reduction by utilizing Local Linear Embedding (LLE) with the inputs of the neighbor subset and the value of dimensionality to be retained. $\mathrm{U} \leftarrow \mathrm{LLE}\left(\mathrm{X}^{\prime}\right), \mathrm{V} \leftarrow \mathrm{LLE}\left(\mathrm{Y}^{\prime}\right)$

d. For every class in Train Dataset construct PLDA models. Four parameters of a model is calculated $(\mu, \Sigma, \mathrm{F}, \mathrm{G})$ : Mean vector of the training data, Covariance matrix, Factor loading matrix of latent identity, Factor loading matrix of latent noise variable(pose-variations). To maximize the log-likelihood function in latent variable model, Expectation-Maximization algorithm is utilized. 
e. Due to the fact that PLDA being a specific form of factor analysis model, different set of parameters will be obtained for different runs. PLDA parameters are taken as average of the specified number of iterations.

f. The 'E- step' computes the expected values of factor loading matrix corresponding to latent identity variables and latent noise variables.

g. In 'M step', there is re-estimation of the conditional distribution of latent variables based on the observed data.

h. During identification PLDA model parameters are used to calculate log- likelihood between enrolled and probe images by utilizing above mentioned EM steps.

$i$. A low value indicates, as usual, that probe image is different from a enrolled identity.

\section{EXPERIMENTAL RESULTS \& THEIR ANALYSIS}

For experimental analysis, train and test database were constructed. Test set comprised of three frontal gallery image and three probe images of twenty subjects with pose variation of up to 45 degrees. Enrolment and Probe dataset is from the AT\&T database [21] while the train data belonged to UMIST database [22]. The training set consisted of ten images each of the twenty subjects (with at least two profile faces).

To extract features from these images, images are processed with Gabor filters (with five scales and eight orientations), and then HVDGP (Gabor pattern) was extracted. Figure 6 shows the instances of Gabor filtered images, and figure 7 shows the Gabor filter bank.
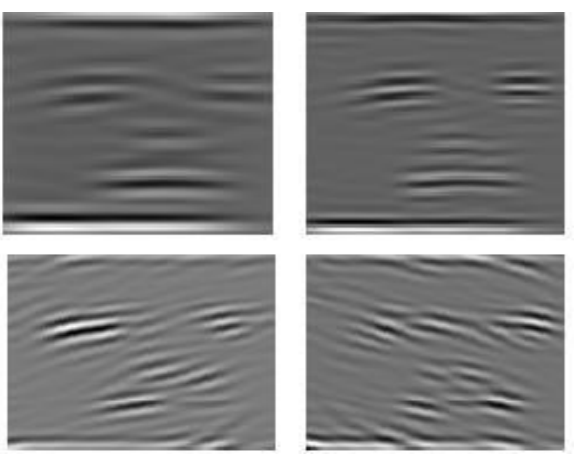

Fig.6. Gabor filtered Image Instances

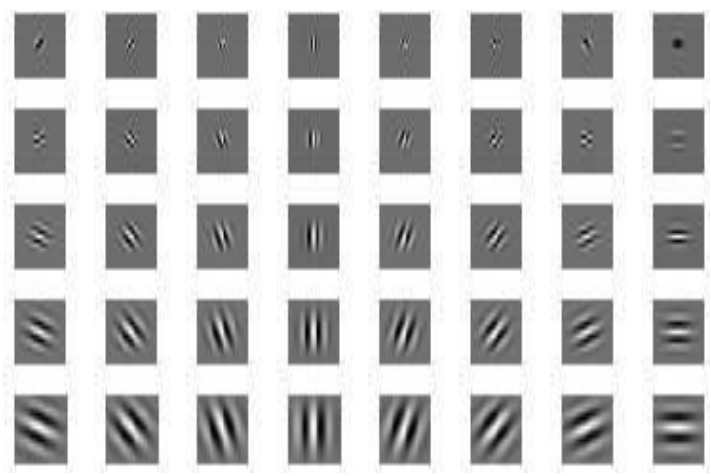

Fig.7. Gabor Filter Bank
The use of quantized binary codes mitigates small variations introduced due to the expression variation or illumination. Gabor magnitude image is depicted in figure $8(\mathrm{a})$ while figure 8 (b) and $8(\mathrm{c})$ are the images obtained by the use of LBP and HVDGBP. From the figures, it can be observed easily that HVDGBP is able to retain all prominent facial features with less noise interference.

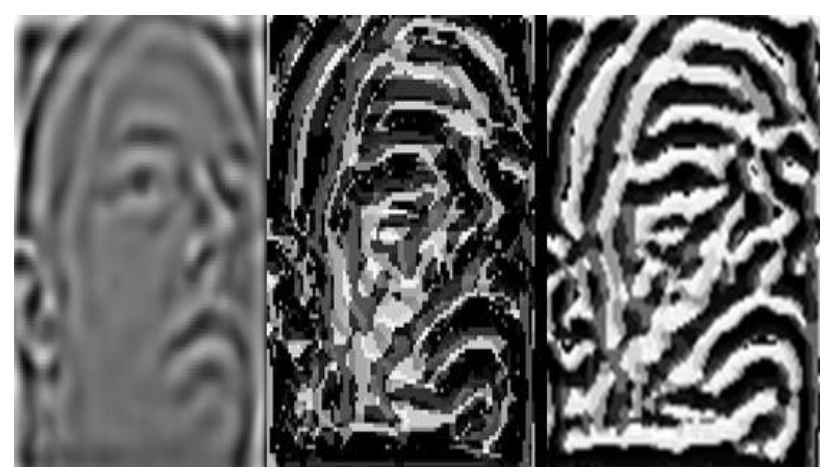

Fig.8(a) Gabor Magnitude Image (b) Gabor-LBP Image (c) GaborHVDBP Image

After HVDGBP feature extraction step, the dimensionality of the feature vector is reduced further by using Locally Linear Embedding Roweis and Saul [25]. Fig.9 shows the dimensionally reduced images with the neighborhood of five and dimensionality retained at one hundred and ten.

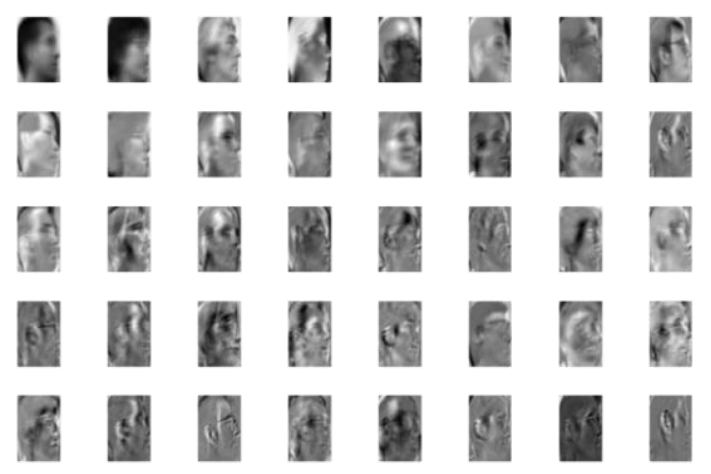

Fig.9. Locally Linear Embedded Images

"Probabilistic Linear Discriminant Analysis" (PLDA) model [16], [19] extends the usage of the idea of latent identity variables (LIVs). PLDA decomposes the face image into additive components: a deterministic component that hinge on fundamental representation of identity exclusively and a stochastic component that describes the pose variations due to which the two face images from the same person are not identical. LIV models unlike subspace algorithms, do not gauge distance measurements between identity representations. Rather they derive identity inference by comparing probe image probabilistic model with gallery's probabilistic models. Statistics of the training data like a zero-order statistic, first-order statistic, and the second-order statistic is computed. Zero-order statistics is global mean and first order statistic is the computation of centralized data. Second order statistic is the covariance of data. Since E 
step involves random number generation, so to generalize the results, fifteen iterations have been taken. During the E step, the first moments of latent variables ( $F \& G$ of eq (8)) are calculated by computing three auxiliary matrices. The rank of $F$ (representing span between individual subspaces) is taken as the number of individuals of the training set, i.e., 20. In $\mathrm{M}$ step, the accumulated second moment of the gathered variables of the first-moment arrays is calculated. Updating of the $F$ and $G$ arrays is done. To speed up the convergence, the minimum divergence step is also applied. PLDA constructs twenty models. The ratio of the log likelihood of the model is calculated for each probe image to determine whether both have the same identity or different identities. Ten iterations are made to overcome the stochastic nature of the model. Twenty PLDA models are constructed. Experiments are performed for identification mode. During identification, a single probability value is calculated for each galley and the enrolled image using PLDA model parameters. A low value indicates, as usual, that each gallery image is from a different gallery identity. Fig. 10 shows the log-likelihood matrix of matching 120 probe images with 120 gallery images. The diagonal elements, to ensure accurate recognition have a higher value than the others.

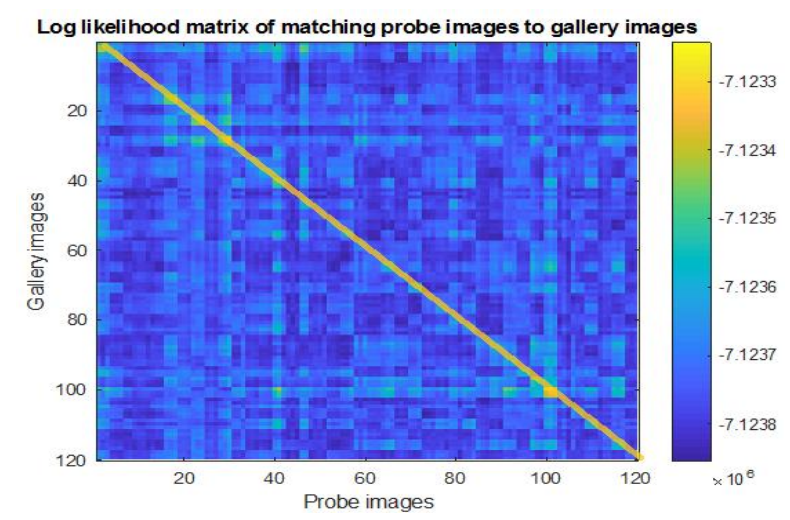

Fig.10. Log Likelihood Matrix of Probe Images vs. Gallery Images

It was also found that the dimensionality of latent variables also affects the recognition rate. Equal Error Rate (EER) which inherently mentions to error margin, a joint point where the false rejections are equal to and false acceptances. Figure 11 shows the equal error rate (EER) vs. the dimensionality of latent variables. EER reduces by increasing the dimensionality of latent variables. Initially, adding dimensions to the latent variables helps in decreasing EER as the information embedded in the added dimensions helps in reducing false acceptance rate. After reaching the value of 100 , further increase in the dimensionality of latent variables does not reduce the EER.

To show the effectiveness of the proposed approach, we compare our approach with existing approaches. For PLDA approach as stated earlier the test set is further divided into gallery set and probe data set. For approaches other than PLDA, test set comprised of both gallery and probe data. Test data comprised of AT\&T database while train data comprised of images from
UMIST database. We compare proposed approach with the basic dimensionality reduction approaches, uniform and non-uniform texture based descriptors, uniform and non-uniform texture based descriptors with dimensionality reduction approaches and PLDA. Table 1 shows the performance of the proposed scheme versus other approaches.

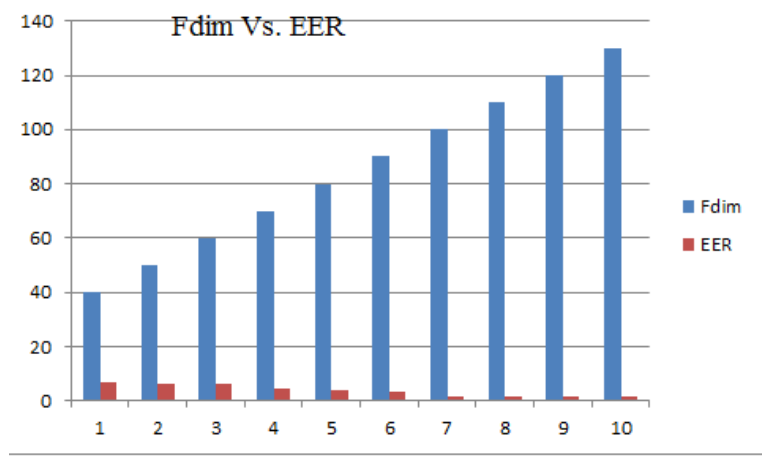

Fig.11. Equal Error Rate for different subspace dimension

Table 1. Recognition Accuracy Comparison

\begin{tabular}{|c|c|}
\hline Approach Used & Average Accuracy(\%) \\
\hline PCA (65) & 56 \\
\hline LDA (65) & 59 \\
\hline LLE(65) & 60.5 \\
\hline KPCA (Polynomial Kernel) & 58.9 \\
\hline PCA+Bayesian (65) & 66 \\
\hline Gabor+LBP & 75 \\
\hline Gabor+LBP+PCA (65) & 76.5 \\
\hline LBP+PCA(65) & 69 \\
\hline LBP+PLDA & 87 \\
\hline LBP+PCA+PLDA & 73.89 \\
\hline HVDGBP+PCA & 92 \\
\hline HVDGBP+KPCA+PLDA & $\mathbf{9 3 . 9 4}$ \\
\hline HVDGBP+LLE+PLDA(*) & \\
\hline Proposed Method &
\end{tabular}

The reason for achieving impressive recognition rate can be attributed to the fact that when the data model is nearly a binary CIA (Conditionally Independent Attribute) model, then EM is able to converge faster and attains optimal performance.

\section{CONCLUSION}

In this paper, we propose the formulation of HVDGBP descriptor and then using PLDA models to find the similarities between identity-related gallery and probe images. While HVDGBP descriptor extracts the local texture details of face images, PLDA models explicitly search for the maximization of the correlated features to retain most informative content between the gallery and probe sets. PLDA attempts to model the difference between the classes of data, so its application helps in achieving better identity recognition even in the presence of pose variations. Since HVDGBP is based on LBP, its 
efficiency is not affected by intensity variations. Though PLDA application gives better identification rates, still it has the issue of retraining score model whenever a new subject is added to the training dataset. For the future works, we will explore other feature descriptors like a bag of words (BOW) with PLDA models.

\section{REFERENCES}

[1] T. Ojala, M. Pietikäinen, and D. Harwood, "A comparative study of texture measures with classification based on feature distributions," Pattern Recognition vol.29 (1) pp. 51-59, 1996. doi:10.1016/00313203(95)00067-4.

[2] T. Ahonen, A. Hadid, and M. Pietikainen, "Face description with local binary patterns: Application to face recognition," IEEE transactions on Pattern Analysis and machine intelligence, vol. 28 (12), pp.2037-2041, 2006.

[3] T. Ahonen, J. Matas, C. He, and M. Pietikäinen, "Rotation invariant image description with local binary pattern histogram fourier features," In Scandinavian Conference on Image Analysis, Springer, Berlin, Heidelberg, 2009.

[4] B. Zhang, Y. Gao, S. Zhao, and J.Liu, "Local derivative pattern versus local binary pattern: face recognition with high-order local pattern descriptor," IEEE transactions on Image Processing, vol.19 (2), pp.533-544, 2010.

[5] J.Trefný, and J.Matas, "Extended set of local binary patterns for rapid object detection," In Computer Vision Winter Workshop, pp. 1-7, 2010.

[6] D.Huang, C.Shan, M. Ardabilian, Y. Wang, and L.Chen, "Local binary patterns and its application to facial image analysis: a survey," IEEE Transactions on Systems, Man, and Cybernetics, Part C (Applications and Reviews), vol. 41(6), pp.765-781, 2011.

[7] S.R. Dubey and S.Mukherjee, "LDOP: Local Directional Order Pattern for Robust Face Retrieval,”,2018. doi: arXiv preprint $: 1803.07441$.

[8] S. ul Hussain, T. Napoleon, and F. Jurie, "Face recognition using local quantized patterns," In $B M V C$, 2012.

[9] L.Liu, S.Lao, P.Fieguth, Y. Guo, X. Wang, and M.Pietikainen, "The Median robust extended local binary pattern for texture classification," IEEE Transactions on Image Processing, vol. 25(3), pp. 1368-1381, 2016.

[10] L.Tian, C.Fan, and Y.Ming, "Learning iterative quantization binary codes for face recognition," Neurocomputing, vol. 214, pp.629-642,2016

[11] J.G. Daugman, Uncertainty relation for resolution in space, spatial frequency, and orientation optimized by twodimensional visual cortical filters, JOSA A, vol. 2(7), pp. 1160-1169, 1985.

[12] Shen, L., Bai, L. and Fairhurst, M., "Gabor wavelets and general discriminant analysis for face identification and verification," Image and Vision Computing, 25(5), pp. 553-563, 2007.

[13] C.A.Perez, L.A. Cament, and L.E. Castillo, "Local matching Gabor entropy weighted face recognition," In Automatic Face \& Gesture Recognition and Workshops, pp.179-184, 2011.

[14] M.Haghighat, S. Zonouz, and M. Abdel-Mottaleb, "Identification Using Encrypted Biometrics," In International Conference on Computer Analysis of Images and Patterns, Springer, Berlin, Heidelberg, pp. 440-448, 2013.
[15] S.K. Jami, S.R. Chalamala, and K.R. Kakkirala, "Cross Local Gabor Binary Pattern Descriptor with Probabilistic Linear Discriminant Analysis for Pose-Invariant Face Recognition," In Computer Modelling \& Simulation (UKSim), UKSim-AMSS 19th International Conference. IEEE, pp. 39-44, 2017.

[16] S.Ioffe, "Probabilistic linear discriminant analysis," In European Conference on Computer Vision, Springer, Berlin, Heidelberg, pp. 531-542 2007.

[17] A.Sizov, K.A.Lee, and T.Kinnunen, "Unifying probabilistic linear discriminant analysis variants in biometric authentication," In Joint IAPR International Workshops on Statistical Techniques in Pattern Recognition (SPR) and Structural and Syntactic Pattern Recognition (SSPR). Springer, Berlin, Heidelberg, pp.464-475, 2014.

[18] S.T.Roweis, and L.K. Saul, "Nonlinear dimensionality reduction by locally linear embedding," science, vol. 290(5500), pp.2323-2326, 2000.

[19] S.J. Prince and J.H. Elder, "Probabilistic linear discriminant analysis for inferences about identity," In Computer Vision, ICCV 2007. IEEE 11th International Conference, pp.1-8, 2007.

[20] S.R. Zhou, J.P. Yin and J.M. Zhang, "Local binary pattern (LBP) and local phase quantization (LBQ) based on Gabor filter for face representation," Neurocomputing, vol. 116, pp.260-264, 2013.

[21] UIMST Face Database; retrieved from: https://www.sheffield.ac.uk/eee/research/iel/research/face

[22] AT\&T-The Database of Faces (formerly "The ORL Database of Faces), retrieved from http://www.cl.cam.ac.uk/research/dtg/attarchive/facedatab ase.html

\section{Authors' Profiles}

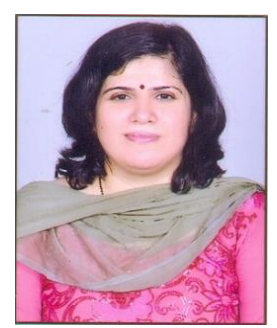

Kumud Arora is Assistant Professor with Inderprastha Enineeerrring College, Ghaziabad since 2007. She is also a parttime research scholar with BanasthaliVidyapeeth, Banasthali, India. She received her M.Tech Degree in Computer Science from IETE, New Delhi in 2014; M.Phil in Computer Science from Alagappa Universty in 2004 and Masters in Computer Application with distinction from Guru Nanak DevUniversity Amritsar, Punjab, India in 1999. She has published research papers in refereed International Journal and Conferences. Her area of research includes digital image processing, machine learning and pattern recognition.

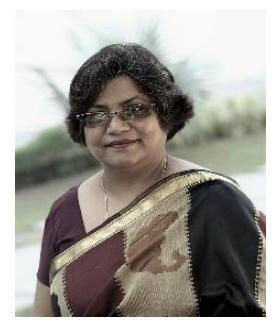

Dr. Poonam Garg is a Professor with Institute of Management Technology, Ghaziabad, India. She did her Ph.d in computer science in 2006 from Banasthali Vidyapeeth, Banasthali. She has $25+$ years of experience in teaching, research and consulting. Her current research interests are in the areas of enterprise resource planning, social media analytics, developing heuristics and meta-heuristics 
particularly genetic algorithm, Tabu search and simulated annealing-based meta-heuristic for various optimization problem such as cryptanalysis of various encryption algorithms and project management. She has published many papers in different International/National journals and has ten edited books. She has served many International Conferences as a conference co-chair, conference track chair and members of program committee. She is also serving as external expert of Doctoral Research Committee for Ph.D programmes at IIS University, MLS University,Indore.

How to cite this paper: Kumud Arora, Poonam Garg, "Horizontal Vertical Diagonal Gabor Binary Pattern Descriptor with PLDA for Pose-Invariant Face Recognition", International Journal of Image, Graphics and Signal Processing(IJIGSP), Vol.10, No.12, pp. 47-55, 2018.DOI: 10.5815/ijigsp.2018.12.06 